\title{
સુરત શહેર અને સુરત ગ્રામ્ય શાળામાં અભ્યાસ કરતા ભાઈઓના શારીરિક અંગઢંગની ખામીઓનો તુલનાત્મક અભ્યાસ
}

\author{
ડ̌.. નિમેશકુમાર ધનસુખભાઇ ચૌધરી
}

\section{પ્રસ્તાવના :}

આજની વિશ્વવ્યાપી અશાંતિના મૂળમાં છે સાચા શિક્ષણની અભાવ. શિક્ષણની જરૂર સમાજને આજ છે તેના કરતાં વધ્ધાર ક્યારે પણ ન હતી. અત્યાર સુધી દુનિયાની સ્થિતિ એવી હતી જ્યારે શિક્ષણની અભાવ પણ માનવના અસ્તિત્વન માટે પડકારરૂપ ન હતો. આજે શિક્ષણ અને તે પણ સાચા શિક્ષણની જરૂર સૌથી વધારે છે, કારણ કે આજે આપણી સમક્ષ સર્વનાશનો ભય ઊભો થયો છે. આ ભય અજ્ઞાનમાંથી ઊભો થયો છે. એ અજ્ઞાન સાચા શિક્ષણના અભાવને આભારી છે.

માનવીના જીવનમાં સૌથી વધારે મહત્વ શરીરનાં હલનચલનનું હોય છે. આપણો બધો જ વ્યવહાર શારીરિક હલનચલન પર જ આધારિત છે. દૈનિક જીવનની ક્રિયાઓમાં મોટાભાગનું શારીરિક હલનચલન હોય છે. શારીરિક હલનચલન સરળ કે જટીલ, ધીમી કે ઝડપી ગતિનું, હળવી કે ભારે પ્રકારનું, ઓછા સમયનુ કे લાંબા સમય સુધી ચાલે ત્યાં સુધીનું હોય, દૈનિક જીવનનું હોય કે વ્યવસાયિક કૌશલ્યો કરવા માટેનું હોય, મનોરંજન હોય કે રમતગમતનાં કૌશલ્યોનું હોય - આ બધા જ પ્રકારના - હલનચલનન સમજવું - અથવા તેના સ્વરૂપને જાણવું આવશ્યક છે. કારણ કે એમ કરવાથી આપણે કોઈપણ કાર્ય પ્રભાવી રૂપથી કરી શકીએ છીએ.

કોઈપણ પ્રકારનું શારીરિક હલનચલન કરવું એનો અર્થ એ નથી થતો કે હલનચલન આપોઆપ પોતાની મેળે જ થાય છે. હલનચલન કરતાં પહેલાં તે બાબતની ચોક્કસ કલ્પના કરવી જરૂરી લેખવામાં આવે છે. ક્રિયા કરવાનો હેતુ, આવશ્યકતા, શરીરના અવયવોનું હલનચલન, ક્રિયાને લાંબા સમય સુધી કરવા માટે શરીરનાં અન્ય આવશ્યક તંત્રોનો સહયોગ, ક્રિયા કરતી વખત તેના પર અસર કરનારાં કેટલાક ભૌતિક-માનસિક પરિબળો વગેરે બાબતોનો અભ્યાસ કરવો જરૂરી છે.

શારીરિક હલનચલનશાસ્ત્રન અંગ્રેજી ભાષામાં કાઈનસિઓલોજી (Kinesiology) કહે છે. માનવીની શારીરિક ક્રિયાઓનું વૈજ્ઞાનક રૂપે અભ્યાસ કરનાંું આ શાસ્ત્ર છે. Kinesiology જે અંગ્રેજી શબ્દ છે, જે ગીક ભાષામાંથી ઉતરી આવ્યો છે. (Kinein) 'કાઈનેઈન' જેનો અર્થ ચાલવું, ફરવું, હાલવું, આગળ જવું (To 
Towards Excellence: An Indexed, Refereed \& Peer Reviewed Journal of Higher Education / Dr.

Nimeshkumar Chaudhari / Page 32-49

Move) વગેરે થાય છે. જ્યારે logesનો અર્થ વિજ્ઞાન અથવા નિબંધ (To discourse). બંને શબ્દોને એક કરીને કાઈનસિઓલોજી કર્યું, જેનો અર્થ માનવ શારીરિક હલનચલન શાસ્ત્ર એમ ધટાવી શકાય છે.

હલનચલનની કુશળતા માટે શરીરની સ્થિતિ હેતુને પૂર્ણ કરનારી અને મહત્વની હોય છે. શરીરન ગતિમાં લાવવા તથા ગતિ ટકાવી રાખવાની દ્રષ્ટિ શરીરની સ્થિતિન એક ખાસ ખૂણામાં રાખવી જરૂરી છે. દોડતી વખતે શરીર આગળની બાજુ ઝૂકેલું રહે છે. વજન ઊંચકતી વખત, શરીરને વજનની નજીક લાવીને જ બળનો ઉપયોગ કરવામાં આવે છે. ઉપરથી તથા દૂરથી આવતા બોલને પકડતી વખત, બોલની દિશામાં શરીરને લાવીન, હથેળીઓન બોલના માર્ગ ઉપર ફેલાવીન પકડવામાં આવે છે. ગોળો ફેંકતા પહેલાં, શરીરને ખાસ સ્થિिિમાં રાખીન ફેંકવા માટે આવશ્યક ગતિ તથા બળનું નિર્માણ કરવા માટેની ક્રિયાની શરૂઆત કરવામાં આવે છે. ક્રિકેટમાં પ્રભાવશાળી બેટિંગ કરવા માટે શરીરની સ્થિતિ તથા શરીરના અવયવોનું હલનચલન મહત્વપૂર્ણ ગણવામાં આવે છે.

અભ्यાસનો હેતુઃ

આ સંશોધન અભ્યાસનો હેતુ “સુરત શહેર અને સુરત ગ્રામ્ય શાળામાં અભ્યાસ કરતા ભાઈઓના શારીરિક અંગઢંગની ખામીઓનો તુલનાત્મક અભ્યાસ” કરવાનો હતો.

વિષયપાત્રોની પસંદગી:

આ સંશોધન અભ્યાસમાં સુરત શહેર શાળામાં અભ્યાસ કરતા ભાઈઓન વિષયપાત્રો તરીકે પસંદ કરવામાં આવ્યા હતા. આ સંશોધન અભ્યાસમાં સુરત ગ્રામ્ય શાળામાં અભ્યાસ કરતા ભાઈઓને વિષયપાત્રો તરીકે પસંદ કરવામાં આવ્યા હતા. આ સંશોધન અભ્યાસમાં 14 થી 16 વર્ષની વય જૂથ ધરાવતા ભાઈઓને વિષયપાત્રો તરીકે પસંદ કરવામાં આવ્યા હતા. આ સંશોધન અભ્યાસમાં સુરત શહેરના 60 ભાઈઓ અને સુરત ગ્રામ્યના 60 ભાઈઓને યદ્ચ્છ પદ્ધતિ દ્વારા પસંદ કરવામાં આવ્યા હતા.

માપનના ધોરણો:

\begin{tabular}{|c|c|c|c|}
\hline ક્રમ & ચલાયમાન & કસોટી & માપન \\
\hline 1 & પગ યાંત્રિકતા કસોટી & \multirow{6}{*}{$\begin{array}{c}\text { આયોવા પોસ્યર } \\
\text { કસોટી }\end{array}$} & \multirow{6}{*}{$\begin{array}{c}\text { આયોવા પોસ્યર } \\
\text { કસોટીના ગુણાંકન } \\
\text { પ્રમાણે }\end{array}$} \\
\hline 2 & ઉભી સ્થિતિ કસોટી & & \\
\hline 3 & ચાલવાની સ્થિતિમાં કસોટી & & \\
\hline 4 & બેઠકની સ્થિતિમાં કસોટી & & \\
\hline 5 & ફલકી વસ્તુ પકડવા માટે વળવાની કસોટી & & \\
\hline 6 & સીડી ઉપર ચઢવાની અને ઉતરવાની કસોટી & & \\
\hline
\end{tabular}


Towards Excellence: An Indexed, Refereed \& Peer Reviewed Journal of Higher Education / Dr.

Nimeshkumar Chaudhari / Page 32-49

કસોટીનું સંચાલન અને આંકડાઓનું એકત્રીકરણ :

આ અભ્યાસમાં આંકડાકીય માહિતી મેળવતા પફેલા બધા જ વિષયપાત્રોને આયોવા પોસ્ચર કસોટી અંગેની વિગતવાર સૂચના આપવામાં આવી હતી. તેમજ કસોટી વહીવટ અને તેના હેતુઓ સમજાવવામાં આવ્યા હતા. ત્યારબાદ કસોટી પ્રયોજવામાં આવી હતી.

૧.) પગ યાંત્રિકતા કસોટીઓ

(ક) ચાલવાની ક્રિયા

- પહેલાં એડીનો જમીનનો સ્પર્શ થવો જોઈએ.

- શરીરનું વજન એડીએથી પગની બહારની ધાર તરફ અને ત્યાંથી પગના બોલ અઅંગૂઠાના પાયા તરફનો સ્નાયુયુક્ત ગોળ ભાગ) તરફ કર્ણની દિશામાં જવું જોઈએ.

- પગનાં આંગળાંનો ઉપયોગ પકડની ક્રિયા માટે થવો જોઈએ.

- ાલવામાં ઉછાળ (Spring) આવવી જોઈએ.

(ખ) સમાંતર પગ બાબતમાં

- પગનાં આંગળાંનો થોડોક બહાર તરફનો ખૂણો (જમણ પગે જમણી બાજુ અને ડાબા પગે ડાબી બાજુ) હોય તે સારી સ્થિતિમાં ગણી શકાય.

- પગનાં આંગળાંનો બફ જ ઓછો અંદર તરફનો ખૂણી (જમણા પગે ડાબી બાજુ અને ડાબા પગે જમણી બાજુ) હોય તો તે સારી સ્થિતિમાં ગણી શકાય.

ગુણાંકન:

જો પગ એક બીજાને સમાંતર રહેતા હોય તો 3 ગુણ, થોડાક જ બહાર તરફ રહેતા હોય તો 2 ગુણ અને વધારે બફાર તરફ રહેતા હોય તો 1 ગુણ આપવામાં આવશે.

૨.)ઊભી સ્થિिિ કસોટી

આ કસોટી માટે વિદ્યાર્થીઓ તેમની ખુરશીઓમાંથી ઊભા થઈન, તેમની ડાબી બાજુની ખુરશીઓ તરફ ઊભા રફેશે. પરીક્ષકો વિદ્યાર્થીઓનું જમણી બાજુથી નિરીક્ષણ કરશે અને જોશે કે વિદ્યાર્થીઓના શરીરના જુદા જુદા ભાગ કેવી સ્થિતિમાં રહે છે અને શરીરનું વજન કેવી રીતે વહેંચાયેલું રહે છે. આ માટે પરીક્ષકો શરીરના જુદા જુદા ભાગોની સ્થિતિઓ નીચેનાં ધોરણોને લક્ષમાં રાખીને જોશે.

- શરીરની કાલ્પનિક ધરી માથું; ડોક, કમર અને બને પગ વચ્ચેથી પસાર થવી જોઈએ.

- માથું અને ડોક ટદાર હોવાં જોઈએ.

- છાતી ઉપર અને આગળ તરફ ખેંચેલી હોવી જોઈએ અને પેટ સપાટ રહેવું જોઈએ.

- ઉપરની ખભા ઉપરની પીઠ સહેજ ગોળાકારે હોય અને પીઠનો નીચેનો ભાગ સફેજ પેટ તરફ ખેંચાયેલો (Slight Hollow) હોય તો તેન સામાન્ય વળાંકમાં ગણવામાં આવશે.

ઊભી સ્થિिિ આરામયુક્ત અને સમતોલનવાળી હોવી જોઈએ. 
Towards Excellence: An Indexed, Refereed \& Peer Reviewed Journal of Higher Education / Dr.

Nimeshkumar Chaudhari / Page 32-49

ગુણાંકન:

સારી ઊભી સ્થિતિ માટે 3 ગુણ, સહેજ સામાન્ય વળાંક માટે 2 ગુણ અને વધારે સામાન્ય વળાંક માટે એક ગુણ આપવામાં આવશ.

3.)ચાલવાની સ્થિતિમાં કસોટી

વિદ્યાર્થીઓ એક બીજાની વચ્ચે 5 થી 6 ફૂટનુ અંતર રાખીને ખુરશીઓની આજુબાજુ ચાલશે. કસોટી લેનાર વિદ્યાર્થીઓની શરીરની સ્થિતિ (Body Alignment) વજનની વહેંચણી, અક્કડપણું અને બિનજરૂરી હલનચલનનું નિરીક્ષણ કરશે. આ નિરીક્ષણમાં નીચેનાં ધોરણો લક્ષમાં રાખશે.

(ક) શરીરના જુદા જુદા ભાગો:

શરીરના જુદા જુદા ભાગોની સ્થિતિનું નિરીક્ષણ ઊભી સ્થિતિ પ્રમાણે કરશે.

ગુણાંકન:

સારી સ્થિતિ માટે 3 ગુણ, સહેજ સામાન્ય વળાંક માટે 2 ગુણ અને વધારે સામાન્ય વળાંક માટે 1 ગુણ આપવામાં આવશે.

(ખ) વજન વહेंચழી:

ચાલવાની સ્થિતિમાં શરીરનું વજન સહેજ આગળ તરફ રહેવું જોઈએ, પરંતુ શરીરની આગળ કે પાછળ તરફનો વળાંક દેખાવો જોઇએ નહીં.

ગુણાંકન:

વજનની સારી અથવા યોગ્ય વહેંચણી માટે 3 ગુણ, થોડાક વળાંક માટે 2 ગુણ અને વધારે વળાંક માટે 1 ગુણ આપવામાં આવશે.

૪.)બેઠકની સ્થિતિમાં કસોટી

વિદ્યાર્થીઓ પહેલાં ખુરશીઓમાં બેસશે. તે સ્થિતિમાં પરીક્ષકી નિરીક્ષણ કરશે. પછી વિદ્યાર્થીઓન બેઠકની સ્થિતિમાંથી આગળ તરફ લગભગ $30^{\circ}$ ના ખૂણે ઝૂકવાનું કહેવામાં આવશે. આ ઝૂકેલી સ્થિતિમાંથી બેઠકની મૂળ સ્થિતિમાં આવ્યા બાદ તેમને ઊભા થવાનું અને થોડાં ડગલાં ચાલવાનું કહેવામાં આવશે. પુનઃ આ બેઠકની અને ચાલવાની સ્થિતિનું નિરીક્ષણ કરવામાં આવશે. પરીક્ષકો ઈચ્છે તો તેમને પુનઃ બેઠકની સ્થિતિ લેવા કહેશે અને નિરીક્ષણ કરશે.

ઉપરોક્ત જુદી જુદી સ્થિતિઓનાં નિરીક્ષણો વખતે નીચેનાં ધોરણો લક્ષમાં લેવાના રહેશે.

(ક) બેઠકની સ્થિતિમા:

- ધડનું સમતોલન કમરના ભાગ ઉપર રહેવું જોઈએ

- ાથું ટદ્ટાર, છાતી ઊંચી અને ખભા પાછળ તરફ (પરંતુ અક્કડતાથી નહીં) રહેવા જોઈએ.

- પેટ સામાન્ય ખેંચેલી સ્થિતિમાં અને પીઠનો ઉપરનો વળાંક સામાન્ય રહેવો જોઈએ.

- પૂંઠ (Hips) નો ભાગ પાછળ તરફ અને ખુરશીના પાછળના ભાગને અડકેલો હોવો જોઈએ. 
Towards Excellence: An Indexed, Refereed \& Peer Reviewed Journal of Higher Education / Dr. Nimeshkumar Chaudhari / Page 32-49

ગુણાંકન:

સાચી સ્થિતિ માટે 3 ગુણ, થોડાક વળાંક માટે 2 ગુણ અને વધારે વળાંક માટે 1 ગુણ આપવામાં आवशे.

(ખ) બેઠકની સ્થિતિમાંથી ઊભા થતાં

- એક પગ થોડોક ખુરશી નીચે, બીજો પગ થોડોક આગળ તરફ, ધડ કમરમાંથી થોડુંક આગળ ઝૂકેલું અને હાથ આરામદાયક સ્થિતિમાં રહેવા જોઈએ.

- પૂં બરાબર શરીર નીચે, પીઠ લગભગ સીધી અને માથું આગળ તરફ ઝૂકવું જોઈએ નહીં.

- ્રિયા સરળ અને આકર્ષક હોવી જોઈએ. સહેજ પણ અક્ક્તા હોવી જોઈશે નહીં.

ગુણાંકન:

સાચી સ્થિતિ માટે 3 ગુણ, ઠીક ઠીક સ્થિતિ માટે 2 ગુણ અને નબળી સ્થિતિ માટે 1 ગુણ આપવામાં आवशे.

૫.)હલકી વસ્તુ પકડવા માટે વળવાની કસોટી

કસોટી માટે વિદ્યાર્થીઓને ખુરશીમાંથી ઊભા થવાનું અને તેમની સામે થોડા અંતરે કોઈ વસ્તુ મુકેલી હશે તેન આગળ વળીને પકડવાનું અને તરત જ તે વસ્તુને જમીન ઉપર મુકવાનું કહેવામાં આવશે. પરીક્ષક આ ક્રિયાઓનું બાજુએથી નિરીક્ષણ કરશે.

વસ્તુને વાળીને પકડવાની ક્રિયા અંગેનાં ધોરણો નીચે પ્રમાણનાં રહેશે.

- વિદ્યાથીઓને વસ્તુ પકડવા માટે ઘૂંટણમાંથી પગને વાળવાના રહેશે. કમરમાંથી શરીર થોડુંક જ આગળ ઝૂકશ.

- ગ અને પૂંઠ શરીરની બરાબર નીચે રહેવા જોઈશ. એક સહેજ આગળ તરફ મૂકવાનો રહેશે.

- ડડભગ ઊભી સ્થિતિમાં જ રહેશે. હાથ મુક્ત રહેશે અને પીઠ કાબૂમાં રહેશે જેથી અક્કડતા આવે નહીં.

- વસ્તુને ઊંચકીને પગની સહેજ આગળ તરફ મૂકવાની રહેશે. ક્રિયા સરળ અને સમતોલનયુક્ત હોવી જોઈशे.

ગુણાંકન:

સારી સ્થિતિ માટે 3 ગુણ, ઠીક સ્થિતि માટે 2 ગુણ અને નબળી સ્થિતિ માટે 1 ગુણ આપવામાં આवशे.

૬.)સીડી ઉપર ચઢવાની અને નીચે ઉતરવાની કસોટી

દરેક વિદ્યાર્થી સીડીનાં 8 થી 10 પગથિયાં ચઢશે અને ઉતરશે. પરીક્ષક બાજુમાં ઊભો રહીને નિરીક્ષણ કરશે અને સીડી ઉપર ચઢવા માટેનું અને સીડી ઉપરથી ઉતરવાનું અલગ અલગ મૂલ્યાંકન કરશે.

(ક) સીડી ઉપર ચઢવા અંગેનાં ધોરણો:

Dec, 2019. VOL.11. ISSUE NO. 3

$\underline{\text { www.hrdcgujarat.ac.in }}$

Page $\mid 36$ 
Towards Excellence: An Indexed, Refereed \& Peer Reviewed Journal of Higher Education / Dr.

Nimeshkumar Chaudhari / Page 32-49

- વજન સહેજ આગળ તરફ રાખવાનું રહેશે, તથા શરીરને કમરમાંથી નહીં પરંતુ ધૂટીમાંથી આગળ તરફ ઝૂકાવવાનું રહેશે.

- ચઢવા માટે ઉપર તરફનો ધક્કો ઘૂંટીમાંથી આપવાનો રહેશે તથા પૂંઠમા ઝલલા ઉત્પન થવા જોઈએ नहीं.

गुણाંકન:

સારી સ્થિતि માટે 3 ગુણ, ઠીક સ્થિતि માટે 2 ગુણ અને નબળી સ્થિતि માટે 1 ગુણ આપવામાં आवशे.

(ખ) સીડી ઉપરથી ઉતરવા અંગેનાં ધોરણો:

- વજન કાબૂ સાથે નીચે તરફ લાવવાનું રહેશે.

- ઉતરવાનું હલનચલન સરળ અને આંચકા (Jerk) વગરનું હોવું જોઈશે.

ગુણાંકન:

સારી સ્થિिિ માટે 3 ગુણ, ઠીક સ્થિતि માટે 2 ગુણ અને નબળી સ્થિતિ માટે 1 ગુણ આપવામાં आवशे.

આંકડાકીય પ્રક્રિયા:

સુરત શહેર અને સુરત ગ્રામ્ય શાળામાં અભ્યાસ કરતા ભાઈઓના શારીરિક અંગઢંગની ખામીઓની તુલના કરવા માટે ' $t$ ' રેશિયો લાગુ પાડી મધ્યકો વચ્ચેના તફાવતીની સાર્થકતા 0.05 કક્ષાએ ચકાસવામાં આवी इતી.

અભ્યાસના પરિણામોઃ

સારણી - 1

પગ યાંત્રિકતા કસોટીમાં ભાઈઓની ચાલવાની ક્રિયાનું 't' રેશિયો દ્વારા પૃથક્કરણ

\begin{tabular}{|c|c|c|c|c|c|}
\hline જૂથ & विषયપાત્રો & મધ્યક & $\begin{array}{c}\text { પ્રમાણ } \\
\text { विચલન }\end{array}$ & મધ્યક તફાવત & ' $t$ ' રેશિયો \\
\hline શહેર & 60 & 2.616 & 0.523 & \multirow{2}{*}{0.033} & \multirow{2}{*}{0.331} \\
\hline ગામ્ય & 60 & 2.65 & 0.577 & & \\
\hline
\end{tabular}

*સાર્થકતાનું ધોરણ 0.05 કક્ષાએ (60)= 1.980

સારણી-1માં ઉપરથી માલુમ પડે છે કે, પગ યાંત્રિકતા કસોટીમાં ચાલવાની ક્રિયાના વિદ્યાર્થી ભાઈઓના મધ્યકો તપાસતા માલુમ પડે છે કે શહેરના વિદ્યાર્થી ભાઈઓનો મધ્યક 2.616 અને ગ્રામ્ય વિદ્યાર્થી ભાઈઓનો મધ્યક 2.65 જોવા મળ્યો હતો. શહેરના વિદ્યાર્થી ભાઈઓનું પ્રમાણિત વિચલન 0.523 અને ગ્રામ્ય વિદ્યાર્થી ભાઈઓનું પ્રમાણિત વિચલન 0.577 જોવા મળ્યું હતું. જેનો મધ્યક તફાવત 0.033 
Towards Excellence: An Indexed, Refereed \& Peer Reviewed Journal of Higher Education / Dr.

Nimeshkumar Chaudhari / Page 32-49

જોવા મज્યો હતો. જેનો ' $t$ ' રેશિયો 0.331 જોવા મળ્યો હતો. જે $0.05(60)=1.980$ કક્ષાએ જોતાં સાર્થક તફાવત જોવા મળ્યો ન ફતો.

માહિતીનું પૃથક્કરણ દર્શાવે છે કે, શહેરમાં અભ્યાસ કરતા ભાઈઓ અન ગ્રામ્યમાં અભ્યાસ કરતા વિદ્યાર્થી ભાઈઓની પગ યાંત્રિકતા કસોટીના ઘટકમાં ચાલવાની ક્રિયા બાબતમાં સાર્થક તફાવત જોવા મળતો ન હતો. બંને જૂથોના વિદ્યાર્થીઓ એકજ જીલ્લામાં રહેતા હોવાથી સમાન વાતાવરણ મળતું હોવાથી બંને જૂથના ભાઈઓમાં ચાલવાની ક્રિયામાં સમાનતા જોવા મળી હતી.

$$
\text { આલેખ - } 1
$$

પગ યાંત્રિકતા કસોટીમાં ભાઈઓની ચાલવાની ક્રિયાના મધ્યક દર્શાવતો આલેખ

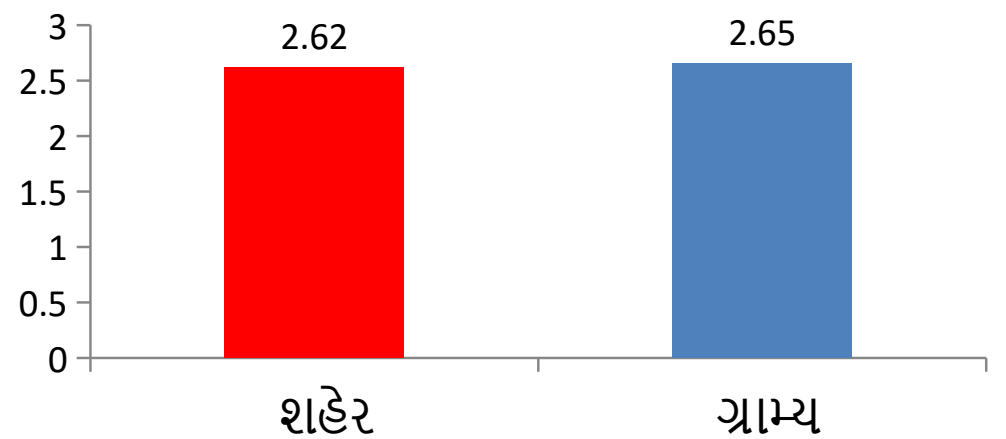

સારણી - 2

પગ યાંત્રિકતા કસોટીમાં ભાઈઓની સમાંતર પગનું 't' રેશિયો દ્વારા પૃથક્કરણ

\begin{tabular}{|c|c|c|c|c|c|}
\hline જૂથ & વિષયપાત્રો & મધ્યક & પ્રમાણ વિચલન & મધ્યક તફાવત & ' $\mathrm{t}$ ' રેશિયો \\
\hline શહેર & 60 & 2.533 & 0.503 & \multirow{2}{*}{0.066} & \multirow{2}{*}{0.732} \\
\cline { 1 - 4 } ગામ્ય & 60 & 2.6 & 0.494 & & \\
\hline
\end{tabular}

*સાર્થકતાનું ધોરણ 0.05 કક્ષાએ $(60)=1.980$

સારણી-2માં ઉપરથી માલુમ પડે છે કે, પગ યાંત્રિકતા કસોટીમાં પગ સમાંતરના વિદ્યાર્થી ભાઈઓના મધ્યકો તપાસતા માલુમ પડે છે કે શહેરના વિદ્યાર્થી ભાઈઓનો મધ્યક 2.533 અને ગામ્ય વિદ્યાર્થી ભાઈઓનો મધ્યક 2.6 જોવા મળ્યો હતી. શહેરના વિદ્યાર્થી ભાઈઓનું પ્રમાણિત વિચલન 0.503 અને ગ્રામ્ય વિદ્યાર્થી ભાઈઓનું પ્રમાણિત વિચલન 0.066 જોવા મળ્યું હતું. જેનો મધ્યક તફાવત 0.066 જોવા મળ્યો હતો. જેનો ' $t$ ' રેશિયો 0.732 જોવા મળ્યો હતો. જે $0.05(60)=1.980$ કક્ષાએ જોતાં સાર્થક તફાવત જોવા મज्यो ન હતો.

માહિતીનું પૃથકકરણ દર્શાવે છે કે, શહેરમાં અભ્યાસ કરતા ભાઈઓ અન ગ્રામ્યમાં અભ્યાસ કરતા વિદ્યાર્થી ભાઈઓની પગ યાંત્રિકતા કસોટીના ધટકમાં સમાંતર પગ બાબતમાં સાર્થક તફાવત જોવા મળતો ન હતો. બંને જૂથોના વિદ્યાર્થીઓ એકજ જીલ્લામાં રહેતા હોવાથી સમાન વાતાવરણ મળતું હોવાથી બંને જૂથના ભાઈઓમાં સમાંતર પગમાં સમાનતા જોવા મળી હતી. 
Towards Excellence: An Indexed, Refereed \& Peer Reviewed Journal of Higher Education / Dr.

Nimeshkumar Chaudhari / Page 32-49

આલેખ - 2

પગ યાંત્રિકતા કસોટીમાં ભાઈઓની સમાંતર પગના મધ્યકો દર્શાવતો આલેખ

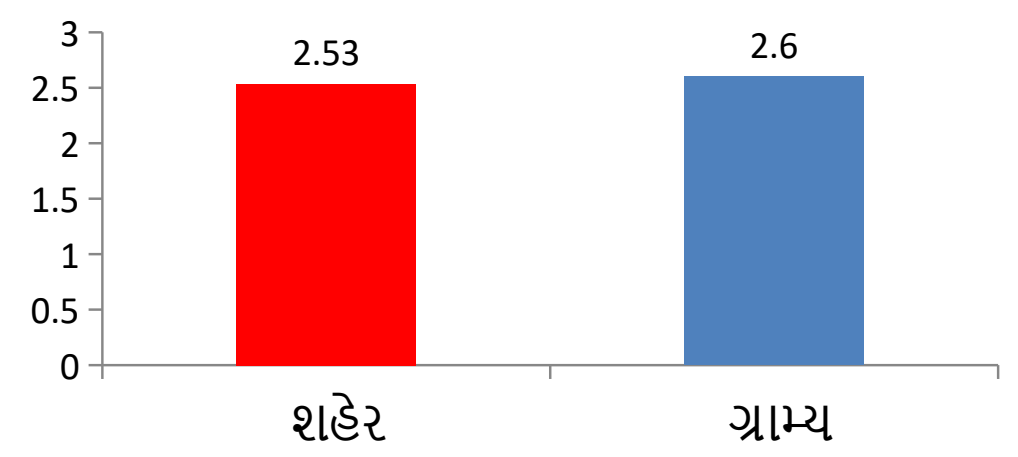

સારણી - 3

ભાઈઓની ઉભી સ્થિતિનું 't' રેશિયો દ્વારા પૃથક્કરણ

\begin{tabular}{|c|c|c|c|c|c|}
\hline જૂથ & वિષયપાત્રો & મધ્યક & પ્રમાણ વિચલન & મધ્યક તફાવત & ' $\mathrm{t}$ ' રેશિયો \\
\hline શહેર & 60 & 2.8 & 0.403 & \multirow{2}{*}{0.216} & \multirow{2}{*}{ 2.62* } \\
\cline { 1 - 4 } ગ્રામ્ય & 60 & 2.583 & 0.497 & & \\
\hline
\end{tabular}

*સાર્થકતાનું ધોરણ 0.05 કક્ષાએ $(60)=1.980$

સારણી-૩માં ઉપરથી માલુમ પડે છે કે, ઉભી સ્થિતિ કસોટીમાં વિદ્યાર્થી ભાઈઓના મધ્યકો તપાસતા માલુમ પડે છે કે શહેરના વિદ્યાર્થી ભાઈઓનો મધ્યક 2.8 અને ગ્રામ્ય વિદ્યાર્થી ભાઈઓનો મધ્યક 2.583 જોવા મળ્યો હતો. શહેરના વિદ્યાર્થી ભાઈઓનું પ્રમાણિત વિચલન 0.403 અને ગામ્ય વિદ્યાર્થી ભાઈઓનું પ્રમાણિત વિચલન 0.497 જોવા મબ્યું હતું. જેનો મધ્યક તફાવત 0.216 જોવા મબ્યો હતો. જેનો ' $t$ ' રેશિયો 2.62 જોવા મબ્યો હતો. જે 0.05 (60) = 1.980 કક્ષાએ જોતાં સાર્થક તફાવત જોવા મળ્યો હતો.

માહિતીનું પૃથક્કરણ દર્શાવે છે કે, શહેરમાં અભ્યાસ કરતા ભાઈઓ અન ગ્રામ્યમાં અભ્યાસ કરતા વિદ્યાર્થી ભાઈઓની ઉભી સ્થિતિ કસોટીમાં સાર્થક તફાવત જોવા મળતો હતો. બંને જૂથના વિદ્યાર્થીઓમાં ખાણી-પીણી અને રહેણીકરણીમાં તફાવત હોવાથી બંને જૂથના ભાઈઓની ભાઈઓની ઉભી સ્થિતિ કસોટીમા સમાનતા જોવા મળી ન હતી. 
Towards Excellence: An Indexed, Refereed \& Peer Reviewed Journal of Higher Education / Dr.

Nimeshkumar Chaudhari / Page 32-49

આલેખ - 3

ભાઈઓની ઉભી સ્થિતિના મધ્યકો દર્શાવતો આલેખ

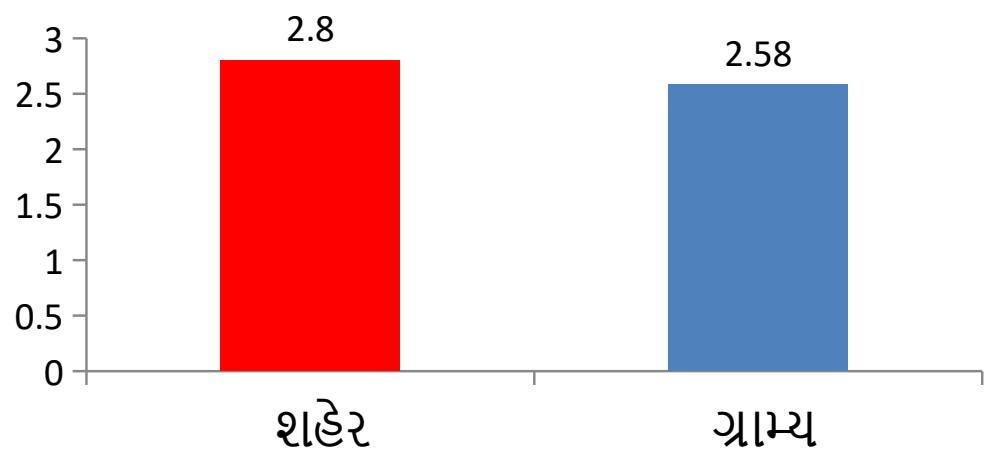

સારણી - 4

ભાઈઓની ચાલવાની સ્થિતિમાં શરીરના જુદા-જુદા ભાગોનું ' $t$ ' રેશિયો દ્વારા પૃથક્કરણ

\begin{tabular}{|c|c|c|c|c|c|}
\hline જૂથ & वિષયપાત્રો & મધ્યક & પ્રમાણ વિચલન & મધ્યક તફાવત & 't' રેશિયો \\
\cline { 1 - 4 } શહેર & 60 & 2.783 & 0.454 & \multirow{2}{*}{0.25} & \multirow{2}{*}{$2.859 *$} \\
\cline { 1 - 4 } ગામ્ય & 60 & 2.533 & 0.503 & & \\
\hline
\end{tabular}

* સાર્થકતાનું ધોરણ 0.05 કક્ષાઓ (60)= 1.980

સારણી-4માં ઉપરથી માલુમ પડે છે કે, ભાઈઓની ચાલવાની સ્થિતિમાં શરીરના જુદા-જુદા ભાગોમાં વજન વહેંચણી કસોટીમાં વિદ્યાર્થી ભાઈઓના મધ્યકો તપાસતા માલુમ પડે છે કે શહેરના વિદ્યાર્थી ભાઈઓનો મધ્યક 2.783 અને ગામ્ય વિદ્યાર્થી ભાઈઓનો મધ્યક 2.533 જોવા મળ્યો હતો. શહેરના વિદ્યાર્થી ભાઈઓનું પ્રમાણિત વિચલન 0.454 અને ગ્રામ્ય વિદ્યાર્થી ભાઈઓનું પ્રમાણિત વિચલન 0.503 જોવા મબ્યું હતું. જેનો મધ્યક તફાવત 0.25 જોવા મબ્યો હતો. જેનો ' $t$ ' રેશિયો 2.859 જોવા મબ્યો હતો. જે 0.05 (60) $=1.980$ કક્ષાઓ જોતાં સાર્થક તફાવત જોવા મબ્યો હતી.

માહિતીનું પૃથક્કરણ દર્શાવે છે કે, શહેરમાં અભ્યાસ કરતા ભાઈઓ અન ગ્રામ્યમાં અભ્યાસ કરતા વિદ્યાર્થી ભાઈઓની ચાલવાની સ્થિતિમાં શરીરના જુદા-જુદા ભાગોમાં વજન વહેંચણી કસોટીમાં સાર્થક તફાવત જોવા મળતો હતો. બંને જૂથના વિદ્યાર્થીઓમાં ખાણી-પીણી અને રહેણીકરણીમાં તફાવત હોવાથી બંને જૂથના ભાઈઓમાં ચાલવાની સ્થિતિમાં શરીરના જુદા-જુદા ભાગોમાં વજન વહેંચણી કસોટીમાં સમાનતા જોવા મળી ન હતી. 
Towards Excellence: An Indexed, Refereed \& Peer Reviewed Journal of Higher Education / Dr.

Nimeshkumar Chaudhari / Page 32-49

આલેખ - 4

ભાઈઓની ચાલવાની સ્થિતિમાં શરીરના જુદા-જુદા ભાગોના મધ્યકો દર્શાવતો આલેખ

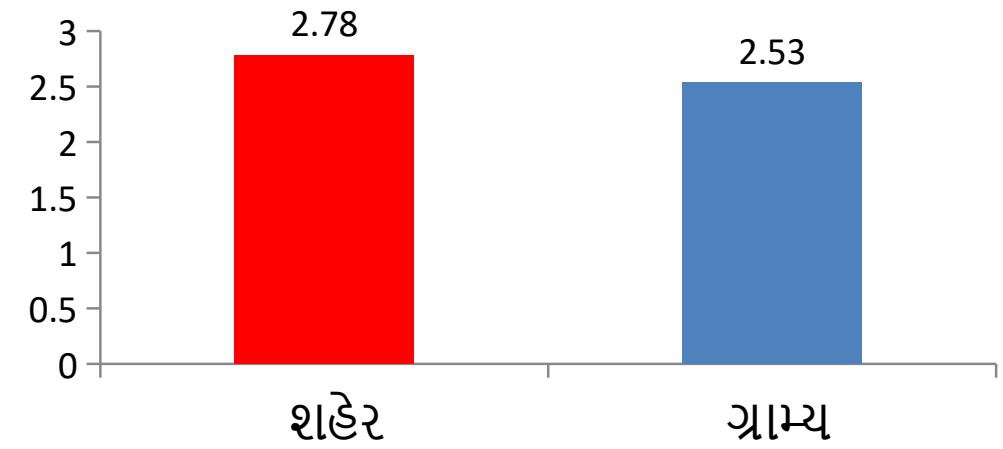

સારણી - 5

ભાઈઓની ચાલવાની સ્થિતિમાં વજન વહેંચણીનું ' $t$ ' રેશિયો દ્વારા પૃથક્કરણ

\begin{tabular}{|c|c|c|c|c|c|}
\hline જૂથ & वિષયપાત્રો & મધ્યક & પ્રમાણ વિચલન & મધ્યક તફાવત & ' $t$ ' રેશિયો \\
\hline શહેર & 60 & 2.6 & 0.494 & \multirow{2}{*}{0.2} & $2.218 *$ \\
\cline { 1 - 3 } ગામ્ય & 60 & 2.4 & 0.494 & & \\
\hline
\end{tabular}

* સાર્થકતાનું ધોરણ 0.05 કક્ષાઓ (60)= 1.980

સારણી-5માં ઉપરથી માલુમ પડે છે કે, ભાઈઓની ચાલવાની સ્થિતિમાં શરીરના જુદા-જુદા ભાગોમાં વજન વહેંચણી કસોટીમાં વિદ્યાર્થી ભાઈઓના મધ્યકો તપાસતા માલુમ પડે છે કે શહેરના વિદ્યાર્થી ભાઈઓનો મધ્યક 2.6 અને ગામ્ય વિદ્યાર્થી ભાઈઓનો મધ્યક 2.4 જોવા મળ્યો હતો. શહેરના વિદ્યાર્થી ભાઈઓનું પ્રમાણિત વિચલન 0.494 અને ગામ્ય વિદ્યાર્થી ભાઈઓનું પ્રમાણિત વિચલન 0.494 જોવા મળ્યું હતું. જેનો મધ્યક તફાવત 0.2 જોવા મબ્યો હતો. જેનો ' $t$ ' રેશિયો 2.218 જોવા મબ્યો હતો. જે 0.05 (60) $=1.980$ કક્ષાઓ જોતાં સાર્થક તફાવત જોવા મળ્યો હતી.

માહિતીનું પૃથક્કરણ દર્શાવે છે કે, શહેરમાં અભ્યાસ કરતા ભાઈઓ અન ગ્રામ્યમાં અભ્યાસ કરતા વિદ્યાર્થી ભાઈઓની ચાલવાની સ્થિતિમાં શરીરના જુદા-જુદા ભાગોમાં વજન વહેંચણી કસોટીમાં સાર્થક તફાવત જોવા મળતો હતો. બંને જૂથના વિદ્યાર્થીઓમાં ખાણી-પીણી અને રહેણીકરણીમાં તફાવત હોવાથી બંને જૂથના ભાઈઓમાં ચાલવાની સ્થિતિમાં શરીરના જુદા-જુદા ભાગોમાં વજન વહેંચણી કસોટીમાં સમાનતા જોવા મળી ન હતી. 
Towards Excellence: An Indexed, Refereed \& Peer Reviewed Journal of Higher Education / Dr.

Nimeshkumar Chaudhari / Page 32-49

$$
\text { આલેખ - } 5
$$

ભાઈઓની ચાલવાની સ્થિતિમાં વજન વહેંચણીના મધ્યકી દર્શાવતો આલેખ

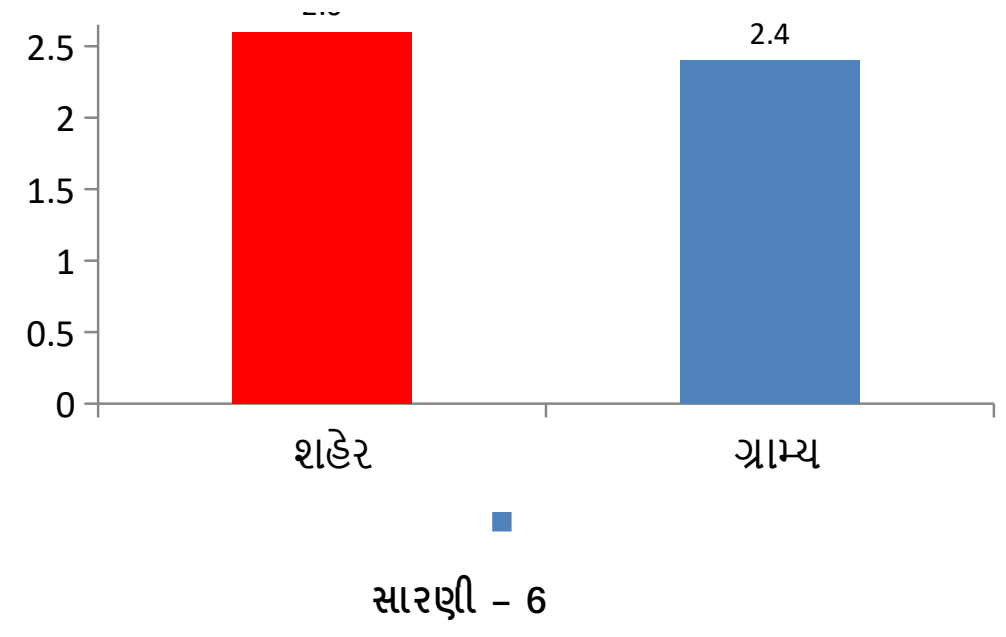

ભાઈઓની બેઠકની સ્થિતિમાં બેઠકની સ્થિતિનું 't' રેશિયો દ્વારા પૃથક્કરણ

\begin{tabular}{|c|c|c|c|c|c|}
\hline જૂથ & वિષયપાત્રો & મધ્યક & પ્રમાણ વિચલન & મધ્યક તફાવત & 't' રેશિયો \\
\cline { 1 - 4 } શહેર & 60 & 2.65 & 0.480 & \multirow{2}{*}{0.083} & 0.932 \\
\cline { 1 - 4 } ગામ્ય & 60 & 2.566 & 0.499 & & \\
\hline
\end{tabular}

* સાર્થકતાનું ધોરણ 0.05 કક્ષાઓ (60)= 1.980

સારણી-6માં ઉપરથી માલુમ પડે છે કે, ભાઈઓની બેઠકની સ્થિતિમાં બેઠકની સ્થિતિ કસોટીમાં વિદ્યાર્થી ભાઈઓના મધ્યકો તપાસતા માલુમ પડે છે કે શહેરના વિદ્યાર્થી ભાઈઓનો મધ્યક 2.65 અને ગ્રામ્ય વિદ્યાર્થી ભાઈઓનો મધ્યક 2.566 જોવા મળ્યો હતો. શહેરના વિદ્યાર્થી ભાઈઓનું પ્રમાણિત વિચલન 0.480 અને ગ્રામ્ય વિદ્યાર્થી ભાઈઓનું પ્રમાણિત વિચલન 0.499 જોવા મબ્યું હતું. જેનો મધ્યક તફાવત 0.083 જોવા મળ્યો હતો. જેનો 't' રેશિયો 0.932 જોવા મળ્યો હતો. જે 0.05 (60) = 1.980 કક્ષાઓ જોતાં સાર્થક તફાવત જોવા મબ્યો ન હતી.

માહિતીનું પૃથક્કરણ દર્શાવે છે કે, શહેરમાં અભ્યાસ કરતા ભાઈઓ અન ગ્રામ્યમાં અભ્યાસ કરતા વિદ્યાર્થી ભાઈઓની બેઠકની સ્થિતિમાં બેઠકની સ્થિતિ કસોટીમાં સાર્થક તફાવત જોવા મળતો ન હતો. બંન જૂથોના વિદ્યાર્થીઓ એક જ જીલ્લામાં રહેતા હોવાથી સમાન વાતાવરણ મળતું હોવાથી બંને જૂથના ભાઈઓની બેઠકની સ્થિતિમાં બેઠકની સ્થિતિ કસોટીમાં સમાનતા જોવા મળી હતી. 
Towards Excellence: An Indexed, Refereed \& Peer Reviewed Journal of Higher Education / Dr.

Nimeshkumar Chaudhari / Page 32-49

આલેખ - 6

ભાઈઓની બેઠકની સ્થિતિમાં બેઠકની સ્થિતિના મધ્યકો દર્શાવતો આલેખ

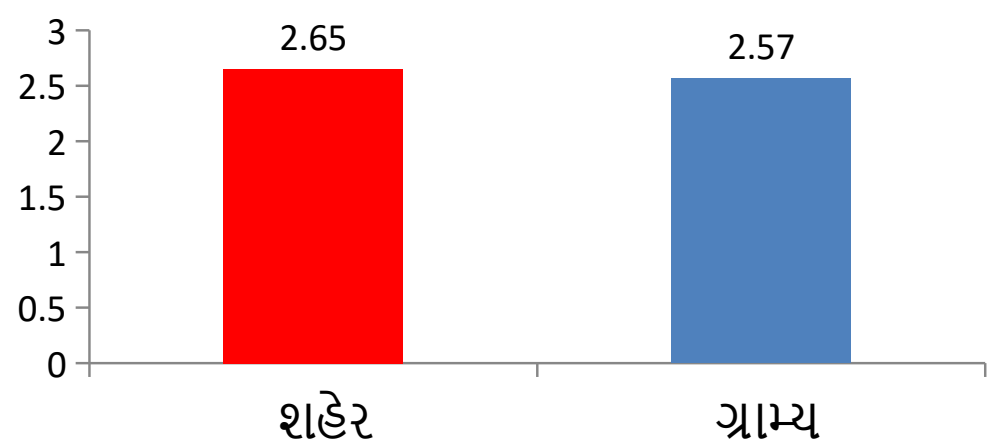

સારણી - 7

ભાઈઓની બેઠકની સ્થિતિમાં ઉભા થતાંની સ્થિતિનું 't' રેશિયો દ્વારા પૃથક્કરણ

\begin{tabular}{|c|c|c|c|c|c|}
\hline જૂથ & वિષયપાત્રો & મધ્યક & પ્રમાણ વિચલન & મધ્યક તફાવત & 't' રેશિયો \\
\cline { 1 - 4 } શહેર & 60 & 2.633 & 0.485 & \multirow{2}{*}{0.266} & \multirow{2}{*}{$3.005 *$} \\
\cline { 1 - 4 } ગામ્ય & 60 & 2.366 & 0.485 & & \\
\hline
\end{tabular}

* સાર્થકતાનું ધોરણ 0.05 કક્ષાઓ (60)= 1.980

સારણી-7 ઉપરથી માલુમ પડે છે કે, ભાઈઓની બેઠકની સ્થિતિમાં ઉભા થતાંની સ્થિતિ કસોટીમાં વિદ્યાર્થી ભાઈઓના મધ્યકો તપાસતા માલુમ પડે છે કે શહેરના વિદ્યાર્થી ભાઈઓનો મધ્યક 2.633 અને ગામ્ય વિદ્યાર્થી ભાઈઓનો મધ્યક 2.366 જોવા મળ્યો હતો. શહેરના વિદ્યાર્થી ભાઈઓનું પ્રમાણિત વિચલન 0.485 અને ગામ્ય વિદ્યાર્થી ભાઈઓનું પ્રમાણિત વિચલન 0.485 જોવા મબ્યું હતું. જેનો મધ્યક તફાવત 0.266 જોવા મળ્યો હતો. જેનો ' $t$ ' રેશિયો 3.005 જોવા મज્યો હતો. જે 0.05 (60) = 1.980 કક્ષાઓ જોતાં સાર્થક તફાવત જોવા મબ્યો હતો.

માહિતીનું પૃથક્કરણ દર્શાવે છે કે, શહેરમાં અભ્યાસ કરતા ભાઈઓ અન ગ્રામ્યમાં અભ્યાસ કરતા વિદ્યાર્થી ભાઈઓની બેઠકની સ્થિતિમાં ઉભા થતાંની સ્થિતિ કસોટીમાં સાર્થક તફાવત જોવા મળતો હતો. બંને જૂથના વિદ્યાર્થીઓમાં ખાણી-પીણી અને રહેણીકરણીમાં તફાવત હોવાથી બંને જૂથના ભાઈઓની બેઠકની સ્થિતિમાં ઉભા થતાંની સ્થિતિ કસોટીમાં સમાનતા જોવા મળી ન હતી. 
Towards Excellence: An Indexed, Refereed \& Peer Reviewed Journal of Higher Education / Dr.

Nimeshkumar Chaudhari / Page 32-49

આલેખ - 7

ભાઈઓની બેઠકની સ્થિતિમાં ઉભા થતાંની સ્થિતિના મધ્યકો દર્શાવતો આલેખ

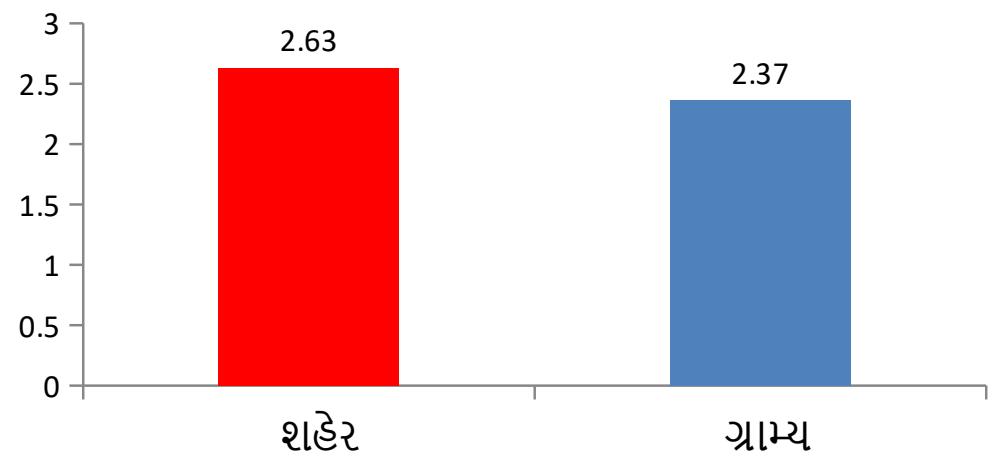

સારણી - 8

ભાઈઓએ જલ્દી વસ્તુ પકડવા માટે વળવાનું 't' રેશિયો દ્વારા પૃથક્કરણ

\begin{tabular}{|c|c|c|c|c|c|}
\hline જૂથ & विષયપાત્રો & મધ્યક & પ્રમાણ વિચલન & મધ્યક તફાવત & 't' રેશિયો \\
\cline { 1 - 4 } શહેર & 60 & 2.65 & 0.480 & \multirow{2}{*}{0.35} & \multirow{2}{*}{$5.638 *$} \\
\cline { 1 - 4 } ગામ્ય & 60 & 3 & 0 & & \\
\hline
\end{tabular}

* સાર્થકતાનું ધોરણ 0.05 કક્ષાઓ (60)= 1.980

સારણી-8 ઉપરથી માલુમ પડે છે કે, ભાઈઓની જલ્દી વસ્તુ પકડવા માટે વળવાનું કસોટીમાં વિદ્યાર્થી ભાઈઓના મધ્યકો તપાસતા માલુમ પડે છે કે શહેરના વિદ્યાર્થી ભાઈઓનો મધ્યક 2.65 અને ગ્રામ્ય વિદ્યાર્થી ભાઈઓનો મધ્યક 3 જોવા મળ્યો હતો. શહેરના વિદ્યાર્થી ભાઈઓનું પ્રમાણિત વિચલન 0.480 અને ગ્રામ્ય વિદ્યાર્થી ભાઈઓનું પ્રમાણિત વિચલન 0 જોવા મળ્યું હતું. જેનો મધ્યક તફાવત 0.35 જોવા મબ્યો હતો. જેનો ' $t$ ' રેશિયો 5.638 જોવા મબ્યો હતો. જે 0.05 (60) = 1.980 કક્ષાઓ જોતાં સાર્થક તફાવત જોવા મબ્યો હતો.

માહિતીનું પૃથક્કરણ દર્શાવે છે કે, શહેરમાં અભ્યાસ કરતા ભાઈઓ અન ગ્રામ્યમાં અભ્યાસ કરતા વિદ્યાર્થી ભાઈઓની જલ્દી વસ્તુ પકડવા માટે વળવાનું કસોટીમાં સાર્થક તફાવત જોવા મળતો હતી. બંન જૂથના વિદ્યાર્થીઓમાં ખાણી-પીણી અને રહેણીકરણીમાં તફાવત હોવાથી બંને જૂથના ભાઈઓની જલ્દી વસ્તુ પકડવા માટે વળવાનું કસોટીમાં સમાનતા જોવા મળી ન હતી. 
Towards Excellence: An Indexed, Refereed \& Peer Reviewed Journal of Higher Education / Dr.

Nimeshkumar Chaudhari / Page 32-49

આલેખ - 8

ભાઈઓએ જલ્દી વસ્તુ પકડવા માટે વળવાના મધ્યકો દર્શાવતો આલેખ

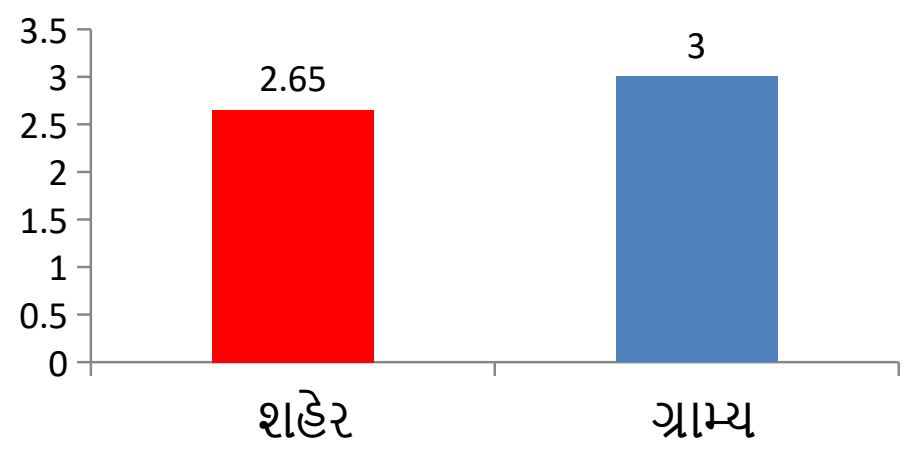

સારણી - 9

ભાઈઓએ સીડી ઉપર ચઢવા અંગેના ધોરણોનું ' $\mathrm{t}$ ' રેશિયો દ્વારા પૃથક્કરણ

\begin{tabular}{|c|c|c|c|c|c|}
\hline જૂથ & વિષયપાત્રો & મધ્યક & પ્રમાણ વિચલન & મધ્યક તફાવત & ' $t$ ' રેશિયો \\
\cline { 1 - 4 } શહેર & 60 & 2.8 & 0.403 & \multirow{2}{*}{0.35} & \multirow{2}{*}{$4.213 *$} \\
\cline { 1 - 4 } ગામ્ય & 60 & 2.45 & 0.501 & & \\
\hline
\end{tabular}

* સાર્થકતાનું ધોરણ 0.05 કક્ષાઓ (60)= 1.980

સારણી-9 ઉપરથી માલુમ પડે છે કે, ભાઈઓની સીડી ઉપર ચઢવા અંગેના ધોરણોનું કસોટીમાં વિદ્યાર્થી ભાઈઓના મધ્યકો તપાસતા માલુમ પડે છે કે શહેરના વિદ્યાર્થી ભાઈઓનો મધ્યક 2.8 અને ગ્રામ્ય વિદ્યાર્થી ભાઈઓનો મધ્યક 2.45 જોવા મળ્યો હતો. શહેરના વિદ્યાર્થી ભાઈઓનું પ્રમાણિત વિચલન 0.403 અને ગામ્ય વિદ્યાર્થી ભાઈઓનું પ્રમાણિત વિચલન 0.501 જોવા મળ્યું હતું. જેનો મધ્યક તફાવત 0.35 જોવા મબ્યો હતો. જેનો ' $t$ ' રેશિયો 4.213 જોવા મળ્યો હતો. જે $0.05(60)=1.980$ કક્ષાઓ જોતાં સાર્થક તફાવત જોવા મબ્યો હતો.

માહિતીનું પૃથક્કરણ દર્શાવે છે કે, શહેરમાં અભ્યાસ કરતા ભાઈઓ અન ગ્રામ્યમાં અભ્યાસ કરતા વિદ્યાર્થી ભાઈઓની સીડી ઉપર ચઢવા અંગેના ધોરણોનું કસોટીમાં સાર્થક તફાવત જોવા મળતો હતો. બંને જૂથના વિદ્યાર્થીઓમાં ખાણી-પીણી અને રહેણીકરણીમાં તફાવત હોવાથી બંને જૂથના ભાઈઓની સીડી ઉપર ચઢવા અંગેના ધોરણોનું કસોટીમાં સમાનતા જોવા મળી ન હતી. 
Towards Excellence: An Indexed, Refereed \& Peer Reviewed Journal of Higher Education / Dr.

Nimeshkumar Chaudhari / Page 32-49

આલેખ - 9

ભાઈઓએ સીડી ઉપર ચઢવા અંગેના ધોરણોના મધ્યકો દર્શાવતો આલેખ

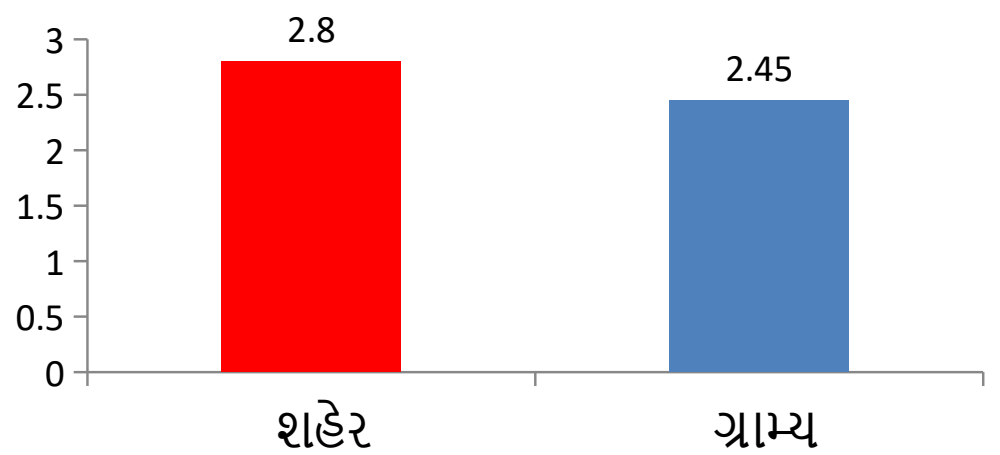

સારણી - 10

ભાઈઓએ સીડી ઉપરથી ઉતરવા અંગેના ધોરણોનું ' $t$ ' રેશિયો દ્વારા પૃથક્કરણ

\begin{tabular}{|c|c|c|c|c|c|}
\hline જૂથ & વિષયપાત્રો & મધ્યક & પ્રમાણ વિચલન & મધ્યક તફાવત & ' $t$ ' રેશિયો \\
\cline { 1 - 4 } શહેર & 60 & 2.55 & 0.501 & \multirow{2}{*}{0.266} & \multirow{2}{*}{$3.049 *$} \\
\cline { 1 - 5 } ગ્રામ્ય & 60 & 2.283 & 0.454 & & \\
\hline
\end{tabular}

*સાર્થકતાનું ધોરણ 0.05 કક્ષાઓ (60)= 1.980

સારણી-10 ઉપરથી માલુમ પડે છે કે, ભાઈઓની સીડી ઉપરથી ઉતરવા અંગેના ધોરણોનું કસોટીમાં વિદ્યાર્થી ભાઈઓના મધ્યકો તપાસતા માલુમ પડે છે કે શહેરના વિદ્યાર્થી ભાઈઓનો મધ્યક 2.55 અને ગ્રામ્ય વિદ્યાર્થી ભાઈઓનો મધ્યક 2.283 જોવા મબ્યો હતો. શહેરના વિદ્યાર્થી ભાઈઓનું પ્રમાણિત વિચલન 0.266 અને ગામ્ય વિદ્યાર્થી ભાઈઓનું પ્રમાણિત વિચલન 0.501 જોવા મબ્યું હતું. જેનો મધ્યક તફાવત 0.454 જોવા મળ્યો હતો. જેનો ' $t$ ' રેશિયો 3.049 જોવા મળ્યો હતો. જે 0.05 (60) = 1.980 કક્ષાઓ જોતાં સાર્થક તફાવત જોવા મબ્યો હતો.

માહિતીનું પૃથક્કરણ દર્શાવે છે કે, શહેરમાં અભ્યાસ કરતા ભાઈઓ અન ગ્રામ્યમાં અભ્યાસ કરતા વિદ્યાર્થી ભાઈઓની સીડી ઉપરથી ઉતરવા અંગેના ધોરણોનું કસોટીમાં સાર્થક તફાવત જોવા મળતો હતો. બંને જૂથના વિદ્યાર્થીઓમાં ખાણી-પીણી અને રહેણીકરણીમાં તફાવત હોવાથી બંને જૂથના ભાઈઓની સીડી ઉપરથી ઉતરવા અંગેના ધોરણોનું કસોટીમાં સમાનતા જોવા મળી ન હતી. 
Towards Excellence: An Indexed, Refereed \& Peer Reviewed Journal of Higher Education / Dr.

Nimeshkumar Chaudhari / Page 32-49

આલેખ - 10

ભાઈઓએ સીડી ઉપરથી ઉતરવા અંગેના ધોરણોના મધ્યકો દર્શાવતી આલેખ

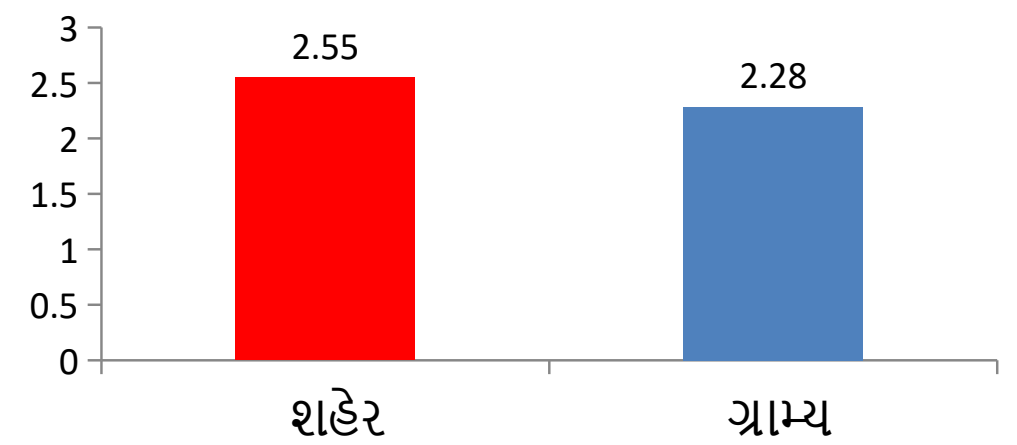

અભ્યાસના તારણો :

- શહેરમાં અભ્યાસ કરતા ભાઈઓ અને ગ્રામ્યમાં અભ્યાસ કરતા વિદ્યાર્થી ભાઈઓની પગ યાંત્રિકતા કસોટીના ઘટકમાં ચાલવાની ક્રિયા બાબતમાં સાર્થક તફાવત જોવા મળતો ન હતો.

- શહેરમાં અભ્યાસ કરતા ભાઈઓ અને ગ્રામ્યમાં અભ્યાસ કરતા વિદ્યાર્થી ભાઈઓની પગ યાંત્રિકતા કસોટીના ઘટકમાં સમાંતર પગ બાબતમાં સાર્થક તફાવત જોવા મળતો ન હતો.

- શહેરમાં અભ્યાસ કરતા ભાઈઓ અને ગ્રામ્યમાં અભ્યાસ કરતા વિદ્યાર્થી ભાઈઓની ઉભી સ્થિતિ કસોટીમાં સાર્થક તફાવત જોવા મળતો હતો.

- શહેરમાં અભ્યાસ કરતા ભાઈઓ અને ગ્રામ્યમાં અભ્યાસ કરતા વિદ્યાર્થી ભાઈઓની ચાલવાની સ્થિતિમાં શરીરના જુદા-જુદા ભાગોમાં વજન વહેંચણી કસોટીમાં સાર્થક તફાવત જોવા મળતો હતો.

- શહેરમાં અભ્યાસ કરતા ભાઈઓ અને ગ્રામ્યમાં અભ્યાસ કરતા વિદ્યાર્થી ભાઈઓની ચાલવાની સ્થિતિમાં શરીરના જુદા-જુદા ભાગોમાં વજન વહેંચણી કસોટીમાં સાર્થક તફાવત જોવા મળતો હતો.

- શહેરમાં અભ્યાસ કરતા ભાઈઓ અને ગ્રામ્યમાં અભ્યાસ કરતા વિદ્યાર્થી ભાઈઓની બેઠકની સ્થિતિમાં બેઠકની સ્થિતિ કસોટીમાં સાર્થક તફાવત જોવા મળતો ન હતો.

- શહેરમાં અભ્યાસ કરતા ભાઈઓ અને ગ્રામ્યમાં અભ્યાસ કરતા વિદ્યાર્થી ભાઈઓની બેઠકની સ્થિતિમાં ઉભા થતાંની સ્થિતિ કસોટીમાં સાર્થક તફાવત જોવા મળતો હતો.

- શહેરમાં અભ્યાસ કરતા ભાઈઓ અને ગ્રામ્યમાં અભ્યાસ કરતા વિદ્યાર્થી ભાઈઓની જલ્દી વસ્તુ પકડવા માટે વળવાનું કસોટીમાં સાર્થક તફાવત જોવા મળતો હતો.

- શહેરમાં અભ્યાસ કરતા ભાઈઓ અને ગ્રામ્યમાં અભ્યાસ કરતા વિદ્યાર્થી ભાઈઓની સીડી ઉપર ચઢવા અંગેના ધોરણોનું કસોટીમાં સાર્થક તફાવત જોવા મળતો હતો. 
Towards Excellence: An Indexed, Refereed \& Peer Reviewed Journal of Higher Education / Dr. Nimeshkumar Chaudhari / Page 32-49

- શહેરમાં અભ્યાસ કરતા ભાઈઓ અને ગ્રામ્યમાં અભ્યાસ કરતા વિદ્યાર્થી ભાઈઓની સીડી ઉપરથી ઉતરવા અંગેના ધોરણોનું કસોટીમાં સાર્થક તફાવત જોવા મળતો હતો.

\section{ભલામણો :}

- આ પ્રકારના સંશોધન કાર્ય જુદી જુદી ઉંમરના વિષયપાત્રો પસંદ કરી ફાથ ધરી શકાય.

- આ પ્રકારના સંશોધન અભ્યાસ માટે વિશાળ નમૂનો લઈને પણ અભ્યાસ હાથ ધરી શકાય.

- આ પ્રકારના સંશોધન અભ્યાસ ભારતના જુદા જુદા ભૌગોલિક વિસ્તારને ધ્યાનમાં રાખીન કરી શકાય.

• આ પ્રકારના સંસોધનો કોલેજોમાં અભ્યાસ કરતા આર્ટસ, કોમર્સ અને સાયન્સ પ્રવાફના વિદ્યાર્થીઓન પસંદ કરી હાથ ધરી શકાય.

• આ પ્રકારના સંશોધનો ગ્રામ્ય અને શહેરી વિસ્તારના વિદ્યાર્થીઓન પસંદ કરી હાથ ધરી શકાય.

- આ પ્રકારના સંશોધનો પ્રાથમિક, માધ્યમિક અને ઉચ્ય માધ્યમિક શાળાના વિદ્યાર્થી ભાઈઓ અને બહેનોને પસંદ કરી ફાથ ધરી શકાય.

• આ પ્રકારના સંશીધનો જુદી જુદી જ્ઞાતિઓના વિષયપાત્રોને પસંદ કરી હાથ ધરી શકાય.

- આ પ્રકારના સંશોધનો જુદી જુદી રમતોના ખેલાડીઓન વિષયપાત્રી તરીકે પસંદ કરી હાથ ધરી શકાય.

- આ પ્રકારનાં સંશોધનો રમતોના જુદા જુદા લેવલે પણ હાથ ધરી શકાય. 
Towards Excellence: An Indexed, Refereed \& Peer Reviewed Journal of Higher Education / Dr. Nimeshkumar Chaudhari / Page 32-49

સંદર્ભસૂચિ :

પટેલ, કાન્તિભાઈ રા. અને કાસુન્દ્રા, પ્રભુલાલ મો., કસોટીઓ માપન અને મૂલ્યાંકન, ગાંધીનગરઃ રમા પ્રકાશન, જુલાઈ, ૨૦૦૧.

પટેલ, હર્ષદભાઈ આઈ. અને અન્યો, શારીરિક હલનચલનશાસ્ત્ર, અમદાવાદ: અનડા બુક ડીપો, ‘ગ્રથસેતુ’, 1756, ગાંધીમાર્ગ, અમદાવાદ-380001.

કાન્ગો ડી., ગડીટી એલ., પીઝા એમ., “રિધમિક જીમ્નાસ્ટીક્સ દ્વારા શરીર શરીર પરિમિતિય પાસાઓનાં વિકાસમાં થતી અસરનો અભ્યાસ”, ઈટાલિયન જર્નલ ઓફ એનેટી એન્ડ એમબોલોજી, જાન્યુઆરી2008સ 113, 1.

રાજપૂત, એલ. જી., “સમાજવિદ્યા વિશારદ અને વ્યાયામ વિશારદના વિદ્યાર્થીઓની શરીરઅંગ પરિમિતિની તુલનાત્મક અભ્યાસ”, અપ્રકાશિત પારંગત પદવી માટેનો લધુનિબંધ, ગૂજરાત વિદ્યાપીઠ, અમદાવાદ, 2003.

\section{ડ̌ı. નિમેશકુમાર ધનસુખભાઇ ચૌધરી ગૂજરાત વિદ્યાપીઠ અમદાવાદ \\ શારીરિક શિક્ષણ અને રમત વિજ્ઞાન વિદ્યાશાખા, સાદરા. તા.જી. ગાંધીનગર. પીન. ૩૮૨૩૨૦}

Email: chaudharinimesh@gmail.com 